\title{
Covert signs of expectancy in serial reaction time tasks revealed by event-related potentials
}

\author{
WERNER SOMMER and HARTMUT LEUTHOLD \\ Humboldt University, Berlin, Germany \\ and \\ ERIC SOETENS \\ University of Brussels, Brussels, Belgium
}

\begin{abstract}
Choice reaction time is strongly determined by the sequence of preceding stimuli. With long response-stimulus intervals (RSIs), a cost-benefit pattern is observed, which has been related to expectancy, whereas with short RSIs a benefit-only pattern emerges, possibly because of automatic facilitation. In the present study, event-related potentials were recorded while subjects performed serial choice responses to visual and auditory stimuli at long and short RSIs. As expected, reaction times displayed cost-benefit and benefit-only patterns at long and short RSIs, respectively. In contrast, sequential effects in event-related potential amplitudes displayed a cost-benefit pattern, unaffected by the RSI. The results demonstrate that an expectancy-like mechanism is always active in serial tasks but appears to influence performance only when the RSI is long.
\end{abstract}

In the present study, we investigate the relation between sequential patterns in reaction times (RTs) and eventrelated potentials (ERPs) in serial two-choice RT experiments. A substantial number of studies have shown that the pattern of sequential effects in RTs changes significantly when the time between the response and the arrival of the next stimulus (response-to-stimulus interval, RSI) is manipulated. With long RSIs, a cost-benefit pattern is observed, suggesting an underlying expectancy-like mechanism, named subjective expectancy (Kirby, 1980). With short RSIs, a benefit-only pattern is observed, suggesting an automatic-like process, named automatic facilitation. Some of the studies with long RSIs have included ERP measurements and have shown that the amplitude of the P300 component displays a cost-benefit pattern that is similar to that for the RTs. The similarity of the cost- benefit patterns strongly indicates that both are elicited by one and the same underlying expectancy mechanism. In the present study, we want to find out whether the parallel between P300 amplitude and RT can be extended to short RSIs and, in general, to contribute to a better understanding of the relative role of expectancy and automatic facilitation mechanisms in sequential effects by recording ERPs.

Many earlier studies have shown that when stimuli from two or more discrete classes are presented in random order, choice RT is strongly determined by the sequence of preceding stimuli (see, e.g., Bertelson, 1963; Kirby, 1976).

This research was supported by the Deutsche Forschungsgemeinschaft (So 177/6-1). Correspondence concerning this article should be sent to W. Sommer, Institut für Psychologie, Humboldt-Universität, Hausvogteiplatz 5-7, 10117 Berlin, Germany (e-mail: werner.sommer@) rz.hu-berlin.de).
The differences in RT are not only visible in the first-order (FO) effect- that is, the influence of the immediately preceding event - but also in higher order (HO) effects, caused by events earlier in the sequence (Kirby, 1976; Soetens, Boer, \& Hueting, 1985). In principle, sequential effects might arise from the sequence of subsequent stimuli, responses, or stimulus-response assignments (Soetens, 1998). Because the present paper is not concerned with the specific locus of the sequential effects within the information processing chain, we prefer neutral terms such as trial sequence or $S-R$ cycle to the more common stimulus sequence.

The sequential pattern of RT changes under various conditions of RSI, compatibility, and type of stimuli used. For example, completely different FO and HO patterns of RTs are observed between conditions with short and long RSIs. These different patterns have been ascribed to two different mechanisms - namely, automatic facilitation for short RSIs and subjective expectancy for long RSIs (see, e.g., Soetens, 1998; Soetens et al., 1985).

With long RSIs - for example, $500 \mathrm{msec}$ or more in a spatially compatible two-choice task-HO sequential effects show a cost-benefit pattern that is typical for expectancy (Audley, 1973). This means that sequences eliciting short RTs when they are followed by a trial repetition (R), proportionally elicit long RTs when followed by a trial alternation (A), and vice versa. For example, when runs of repetitions (RRR) or alternations (AAA) are continued (RRRR, AAAA), RT decreases with the length of the run, whereas RT increases if the same runs are interrupted ( $\mathrm{RRR} A, \mathrm{AAAR}$ ). In the first case, $\mathrm{RT}$ is assumed to be fast because the actual event matches the expectancy of the subjects, whereas, in the second case, RT is long because expectancy is disconfirmed. 
In contrast to long RSIs, no cost-benefit pattern emerges in two-choice tasks when RSIs are short-for example, $100 \mathrm{msec}$ or less. On the contrary, some sequences then only have benefits and are always followed by short RTs (e.g., RRR $R, \operatorname{RRR} A$ ), whereas other sequences are followed by long RTs (e.g., AAA $A$, AAA $R$ ). This is called a benefit-only pattern and has been associated with the concept of automatic facilitation (Kirby, 1976; Soetens, Deboeck, \& Hueting, 1984; Vervaeck \& Boer, 1980). Automatic facilitation explains the benefit-only pattern as being a consequence of residual memory traces left by previous stimulus-response cycles, decaying over time (Bertelson, 1961; Hyman, 1953). Kirby (1980) suggests that, with short RSIs, these traces have not faded out by the arrival of the next stimulus, so that HO effects can ensue. However, the results of many studies show that the passage of time might not be the only determining factor, but also the number of intervening stimuli (Pashler \& Baylis, 1991; Soetens, 1990, p. 62). Thus, it is possible that any remaining traces of previous stimulus-response cycles in the RT pattern are hidden by the upcoming influence of subjective expectancy with increasing RSIs.

Automatic facilitation differs from typical repetition priming effects in that it is not event specific. That is, some sequences seem to create a state of the organism that is beneficial for the processing of any stimulus that will come next, whereas other sequences create a detrimental state. Laming (1968) described the effect as being noise in the process of identifying the stimulus, which increases when many stimulus alternations have occurred, whereas it decreases with many preceding repetitions.

Also, stimulus-response compatibility and the type of stimuli used influence the specific RSI at which a benefit-only pattern changes into a cost-benefit pattern. For example, repetition effects or benefit-only patterns at long RSIs have been found with symbolic stimuli or when more than two response alternatives are used (see, e.g., Bertelson \& Renkin, 1966; Campbell \& Proctor, 1993; Kirby, 1980; Pashler \& Baylis, 1991). Although the switch to a cost-benefit pattern in spatially compatible two-choice situations has been observed at RSIs of $100 \mathrm{msec}$, much longer RSIs are necessary for the change in pattern in less compatible conditions (Soetens et al., 1985).

The cost-benefit (expectancy) and benefit-only (automatic facilitation) terminology suggests that reference should be made to the theory of controlled and automatic processing (Posner \& Snyder, 1975; Schneider \& Shiffrin, 1977; Shiffrin \& Schneider, 1977). However, the term expectancy in the present context is mainly used to describe a phenomenon that elicits a cost-benefit pattern in RTs or ERPs and does not necessarily refer to voluntary, conscious, or controlled processes. Whether or not these attributes hold for the sequence-dependent "expectancies" is an empirical question. We come back to this issue in the general discussion.

Although, in some conditions, HO sequential effects are dominated by automatic facilitation and in others by expectancy, the relationship between both processes remains to be determined. That expectancies may be present even at short RSIs, where a benefit-only pattern is normally dominant, is suggested by the findings of Soetens et al. (1985; Soetens et al., 1984). After long alternation sequences, RTs were unusually short when they were continued (AAAA), and RTs were unusually long when they were discontinued (AAAR). That is, such sequences formed an exceptional cost-benefit pattern with short RSIs in an otherwise dominating benefitonly pattern of automatic facilitation. It seems as if, after a highly conspicuous series of stimuli, expectancy suddenly breaks through. Soetens et al. (1985) argued that a differentiation should be made between expectancy, on the one hand, and observable effects of these expectancies on behavior as a consequence of preparation, on the other hand. Expectancies may be present in all conditions, but with short RSIs, they may not influence behavior because not enough time is available to prepare responses on the basis of these expectancies.

One way to approach the question of whether there are covert expectancy processes when in overt behavior a benefit-only is observed is the use of event-related brain potentials. ERPs consist of various components that can be related to the ongoing activity of different aspects of information processing and can be an indication of covert processes. In relation to the present questions, it is possible that RTs and ERPs follow different patterns in short-RSI conditions.

Patterns of ERPs in serial choice RTs have not yet been studied with short-RSI conditions, but a lot of research has been done with long RSIs. In ERP studies with relatively long and constant interstimulus intervals (ISIs), sequential effects similar to those with RTs at long RSIs have been observed also in the amplitude of the P300 component when large amplitudes are considered to reflect more costly processing than do small ones. For example, Squires, Wickens, Squires, and Donchin (1976) found that sequential effects in P300 amplitude followed a cost-benefit pattern, indicative of an expectancy mechanism. Many subsequent researchers have replicated the similarity of sequential effects of P300 amplitude and response latencies in choice RT tasks (Duncan-Johnson, Roth, \& Kopell, 1984; Ford, Duncan-Johnson, Pfefferbaum, \& Kopell, 1982; Sommer, Matt, \& Leuthold, 1990a). This is considered to be strong evidence that the pattern of changes in $\mathrm{P} 300$ amplitude and RTs is elicited by one and the same expectancy mechanism.

However, recent research has shown that sequencedependent RT and ERP patterns for the same data set may be dissociated. For relatively long ISIs of $1.3 \mathrm{sec}$, Sommer et al. (1990a) showed an FO alternation effect - that is, RTs were faster to a different stimulus than to the same stimulus. P300 amplitude, on the other hand, displayed an FO repetition effect-that is, the amplitude was smaller for repeated than for unrepeated stimuli. The FO effects in P300 amplitude and in RT become more similar with 
practice (Sommer, Matt, \& Leuthold, 1990b) and with further increasing ISIs (Leuthold \& Sommer, 1993).

Important for the present purposes is that the HO sequential effects in P300 amplitude cannot be ascribed to a strictly controllable process (cf. Sommer, Leuthold, \& Matt, 1998). Matt, Leuthold, and Sommer (1992) manipulated voluntary expectancies of their subjects toward a preference for stimulus alternations or repetitions, whereas in fact they were equiprobable. Although there were clear alterations of sequential effects in RTs, changes for P300 amplitude were few and small. Also, Sommer et al. (1990a) showed that conscious expectancies for a given stimulus, as measured with subjective ratings, are hardly affected by the trial sequence at all. Finally, HO sequential effects in $\mathrm{P} 300$ amplitude are unaffected by even large amounts of practice (Sommer et al., 1990b). On the basis of their results, Sommer et al. (1998) suggested that sequential effects in P300 are generated by a lowlevel and largely uncontrollable variant of expectancy.

If the expectancy mechanism is uncontrollable, it might also remain active at short RSIs. It is conceivable, then, that P300 amplitude still shows a cost-benefit pattern even when RTs show a relatively pure benefit-only pattern. If, on the other hand, there is no time to develop expectancies in short-RSI conditions (Vervaeck \& Boer, 1980), the cost-benefit pattern in P300 amplitude should not be present. In this paper, we investigate this hypothesis in two experiments using two-choice stimulus location to response location tasks, in the auditory and visual modality, respectively.

\section{EXPERIMENT 1}

\section{Method}

Subjects. We tested a total of 10 right-handed subjects ( 6 males), who were from 22 to 35 years of age. They had no prior experience in the particular task of this study.

Apparatus and Stimuli. Stimuli consisted of single $40-\mathrm{msec}$ bursts of $66-\mathrm{dB}(\mathrm{A})$ white noise, presented at random and equiprobably to either ear via headphones. RSI, defined as the interval between response onset and onset of the next stimulus, was either 40 or $500 \mathrm{msec}$. Coulburn modules controlled stimulus presentation. Stimuli and responses were coded as voltage pulses for a trigger signal. Responses were performed on two telegraph keys, mounted $20 \mathrm{~cm}$ apart on a horizontal panel, orthogonal to the midsaggital plane. The keys were operated with the index fingers.

Procedure. The subjects were comfortably seated in a dimly lit, sound attenuated, and electrically shieided room, using a fixed chin rest. They responded compatibly to right- and left-ear stimuli, respectively, with right and left keypresses, which were to be both fast and accurate.

The subjects were advised to fixate a dot in front while responding. The 40- and 500-msec RSI conditions lasted about 20 and $40 \mathrm{~min}$, interrupted by short breaks after every 50 and $100 \mathrm{sec}$, respectively. Each subject participated first in the 40 - and then in the 500 -msec RSI condition. The order of the RSI conditions was not balanced, because HO sequence effects in RTs are much less affected by practice at long RSIs than by practice at short RSIs (Soetens et al., 1985; Soetens et al., 1984). Similarly, at long RSIs, HO sequence effects in $\mathrm{P} 300$ amplitude are practice insensitive (Sommer et al., 1990b).
Electrophysiological recordings. The electroencephalogram (EEG) was recorded with a linked mastoid reference from $\mathrm{Fz}, \mathrm{Cz}$, and $\mathrm{Pz}(\mathrm{TC}=10 \mathrm{sec}, \mathrm{HFF}=40 \mathrm{~Hz})$. In addition, the vertical electrooculogram (vEOG) was recorded from electrodes above and below the right eye. These electrophysiological signals were digitized along with the trigger signal at $100 \mathrm{~Hz}$ for continuous time segments of $18.5 \mathrm{sec}$. Data storage between segments required less than $1.5 \mathrm{sec}$. Off line, the trigger signals were decoded, and the data were separated into individual trials with 400 -msec prestimulus and 690 -msec poststimulus epochs.

Data analyses. Each trial was coded according to 16 fourthorder sequences of trial repetitions and alternations (from RRRR over AAAR and RRRA to AAAA). Analyses of RTs, error rates, and ERPs were based on these sequences. The same set of trials was considered for both RTs and ERPs. In these trials, both the present and the immediately preceding response had to be correct, and the respective ERPs had to be artifact free. A response was considered correct if the proper key had been pressed no earlier than $150 \mathrm{msec}$ following stimulus onset. For the error rates, only present trials were considered, and any artifacts in ERPs from eye movements, blinks, or amplifier saturation were disregarded. The number of artifacts did not vary as a function of experimental condition. Note that errors and artifacts did not affect the classification of the trial sequence.

\section{Results and Discussion}

Performance. To check the influence of RSI on HO effects, we used the slopes of the repetition and alternation functions, as described by Soetens et al. (1985). Therefore, RTs are depicted in Figure 1 as a function of $\mathrm{HO}$ sequences for both RSI conditions. The left part of the curve, representing all sequences ending with an FO repetition, is called the repetition curve. The right part is

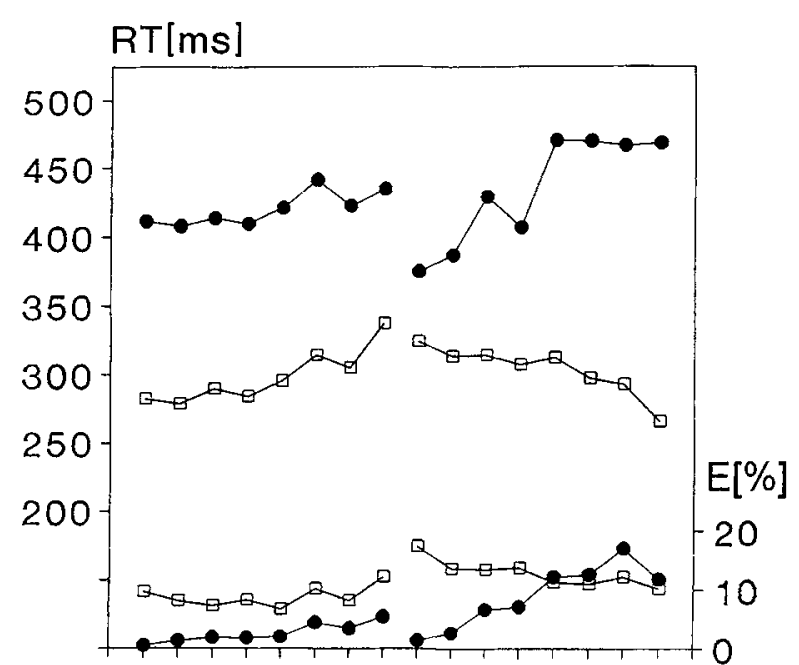

RARARARARARARARA RRAARRAARRAARRAA RRRRAAAARRRRAAAA RRRRRRRRAAAAAAAA

$\because$ RSI $40 \mathrm{~ms}-\mathrm{RSI} 500 \mathrm{~ms}$

Figure 1. Experiment 1: Sequential effects in reaction times and error rates. 
called the alternation curve, as it contains all sequences ending with an FO alternation. The critical parameter is the slope of the alternation function. A positive slope, parallel to the repetition curve, is a benefit-only pattern, typical for automatic facilitation, whereas a negative slope, opposite to the slope of the repetition curve, is a costbenefit pattern, typical for subjective expectancy. In the following analyses, differences in the slopes are assessed by comparing the linear components of the regressions lines for $\mathrm{FO}$ repetition and alternation sequences.

An analysis of variance (ANOVA) was performed on RT with repeated measures on factors RSI (RSI 40 vs. RSI 500), FO sequence (repetitions vs. alternations), and HO sequences (RRR to AAA).

Responses were faster in the RSI 500 than in the RSI 40 condition $[M=301$ vs. $428 \mathrm{msec}, F(1,9)=23.5, p<$ $.001]$. There was no FO effect, either in the overall data or in any of the RSI conditions. However, there was a strong $\mathrm{RSI} \times \mathrm{FO} \times \mathrm{HO}$ interaction $[F(1,9)=28.1, p<.001]$. For the RSI 40 condition, the slopes of both the repetition and the alternation branches are positive, but more so for the alternation branch $[F(1,9)=9.0, p<.05]$. In contrast, the linear trends across the repetition and alternation branches in the RSI 500 condition differed in direction $[F(1,9)=46.5, p<.001]$. A different way of looking at the RSI $\times \mathrm{FO} \times \mathrm{HO}$ interaction is by comparing trends across RSI conditions, separately for repetition and alternation branches. The slopes of the repetition curves are positive for both RSIs, yielding a main effect for the linear trend across the $\mathrm{HO}$ sequences $[F(1,9)=71.9, p<.001]$. The slight difference in the positive slopes was reflected by a tendency for an RSI $\times$ HO interaction $(p=.07)$. The linear trends across the alternation branches of the RSI conditions differed in direction $[F(1,9)=31.6, p<$ $.001]$. The positive RSI 40 slope was steeper than the negative RSI 500 slope, yielding a small main effect of the HO sequence $[F(1,9)=8.8, p<.05]$.

More errors were made for long RSIs than for short RSIs $[M=10.8 \%$ vs. $5.8 \% ; F(1,9)=9.3, p<.05]$. In line with predictions, more errors were made for sequences ending with an alternation than for those ending with repetitions $[M=10.9 \%$ vs. $5.7 \% ; F(1,9)=15.5, p<.01]$, and this was so for short- as well as for long-RSI conditions. Sequential effects in error rates involving $\mathrm{HO}$ sequences are similar to the pattern in mean RTs, as can be seen in Figure 1. All error curves are parallel to RT curves. This similarity in pattern between RTs and error rates excludes an explanation of the sequential effects in terms of a speed-accuracy tradeoff.

In particular, the opposite slopes of the alternation curve were replicated in the error data. As was the case for RTs, there was an RSI $\times \mathrm{FO} \times \mathrm{HO}$ interaction $[F(1,9)=11.1$, $p<.01]$, again stressing the different patterns for both RSI conditions. This latter interaction was evidently caused by a difference in the HO slope across the alternation branch between the RSI conditions $[F(1,9)=19.3, p<$ $.01]$, which was not present for the repetition branch $(F=1.1)$.
On the whole, the two conditions of this study show two different sequential RT patterns. They reproduce RT patterns, similar to those described in earlier studies (Kirby, 1976; Soetens et al., 1985), that have been related to different mechanisms, named automatic facilitation and subjective expectancy. The present study extends these findings from visual to auditory stimuli. The repeated measures design and fixed ordering for the RSI conditions may have led to an increased error rate in the RSI 500 condition, as compared with the findings of Soetens et al. (1985), but do not seem to have interfered with the HO sequential patterns.

P300 amplitude. For the RSI 40 condition, there was considerable overlap of the ERPs between successive trials. This is shown in the left panel of Figure 2 for the ERPs at each electrode of 1 subject and for some selected trial sequences. The overlap can be seen most clearly in the prestimulus time segments for which there is systematic ERP activity. For example, in the RRAR sequence, we see a clear positive peak around $200 \mathrm{msec}$ prior to stimulus onset, followed by a smaller negative peak. This overlap most likely extends also into the poststimulus segment. Such overlap between subsequent ERPs not only distorts the measurement of the component in question, but also makes it virtually impossible to find a suitable baseline. Overlap from ERPs to subsequent stimuli, on the other hand, should generally be less problematic, because, with RTs of more than 400 msec, the next P300 should come late enough not to distort the present P300. We therefore applied the procedure for the correction of overlap between adjacent ERPs (ADJAR), developed by Woldorff (1993), to the ERPs of the RSI 40 condition before making any amplitude measurements. ${ }^{1}$

In its advanced version (Level 2), ADJAR yields separate estimates of the contributions of the ERPs elicited by the preceding and the subsequent stimuli to any given ERP. In an iterative procedure, these estimates are optimized, and, finally, both are subtracted from the original ERP. We ran this procedure with 10 iterations where all estimates had approached asymptote. The right panel of Figure 2 demonstrates the resulting waveform. After ADJAR, the prestimulus epochs showed considerably less activity, and the final part of the recording epochs showed a better return to baseline than before correction. Figure 3, left panel, shows the grand average of the ADJAR-corrected ERPs from the RSI 40 condition. Note that, even after ADJAR, there was some systematic activity starting about $100 \mathrm{msec}$ preceding the stimulus. Clearly, this must be response-related activity to the previous stimulus, which is time-locked to the stimulus eliciting the present ERP by our use of constant RSIs. Because ADJAR cannot remove this stimulus-locked ERP activity, we used a baseline from 400 to $200 \mathrm{msec}$ preceding the stimulus for the measurement of all ERP amplitudes.

Figure 3 clearly demonstrates cost-benefit patterns when the ERPs for continued and interrupted runs of both repetitions and alternations are compared. Large amplitudes are recorded after unexpected sequences- 


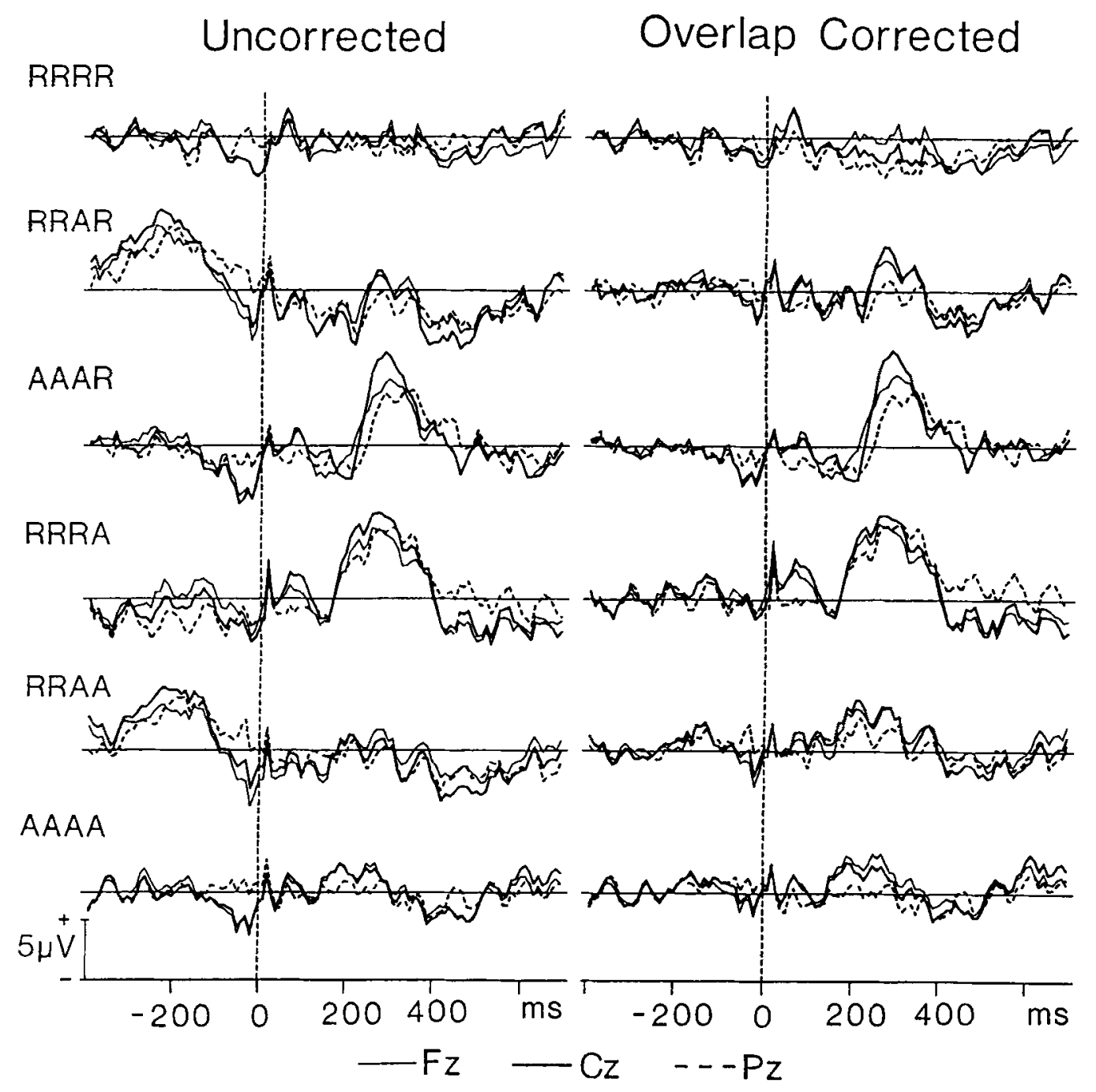

Figure 2. Experiment 1: Demonstration of the effect of overlap correction for the event-related potentials (ERPs) of 1 subject from the RSI 40 condition. The figure displays ERPs of the most extreme and also of two intermediate sequences (RRAR and RRAA) in its original (left column) and corrected versions (right column). Note the smoothening of the prestimulus baselines, particularly for the RRAR and RRAA sequences.

that is, interruptions of runs - whereas small amplitudes are recorded after expected sequences-that is, continuations of runs. This pattern holds not only for the RSI 500 condition, where it was expected, but also for the RSI 40 condition.

P300 amplitude was quantified as average amplitude in an interval between 280 and $370 \mathrm{msec}$ - centered around the average peak latency-relative to the baseline just described. Figure 4 displays the sequential effects in this measure for both RSIs. The figures confirm the costbenefit pattern for the HO sequences. The slopes of the repetition curves are positive, and the slopes of the alternation curves are negative. This pattern is in agreement with the pattern of RTs for the RSI 500 condition but is opposite to the RT pattern in the RSI 40 condition. Also, the commonly observed FO repetition effect for both
RSI conditions is visible as the difference between the general levels of the repetition and alternation curves.

The same ANOVAs as those used for the RT data were applied to P300 amplitude, with the additional inclusion of repeated measures on electrode site $(\mathrm{Fz}, \mathrm{Cz}, \mathrm{Pz})$. In some cases, the question of differential scalp distributions across experimental conditions arises. Here an ambiguity may emerge when there are overall amplitude differences between conditions (McCarthy \& Wood, 1985). When amplitude changes occur because of variations in the activity of an invariant underlying intracranial generator structure, the effects at different electrodes will be multiplicative-for example, increasing or decreasing the amplitude by a certain percentage at each electrode. In this case, because of its additive model, the ANOVA will yield an interaction between the condition in question 


\section{RSI $40 \mathrm{~ms} \quad$ RSI $500 \mathrm{~ms}$}
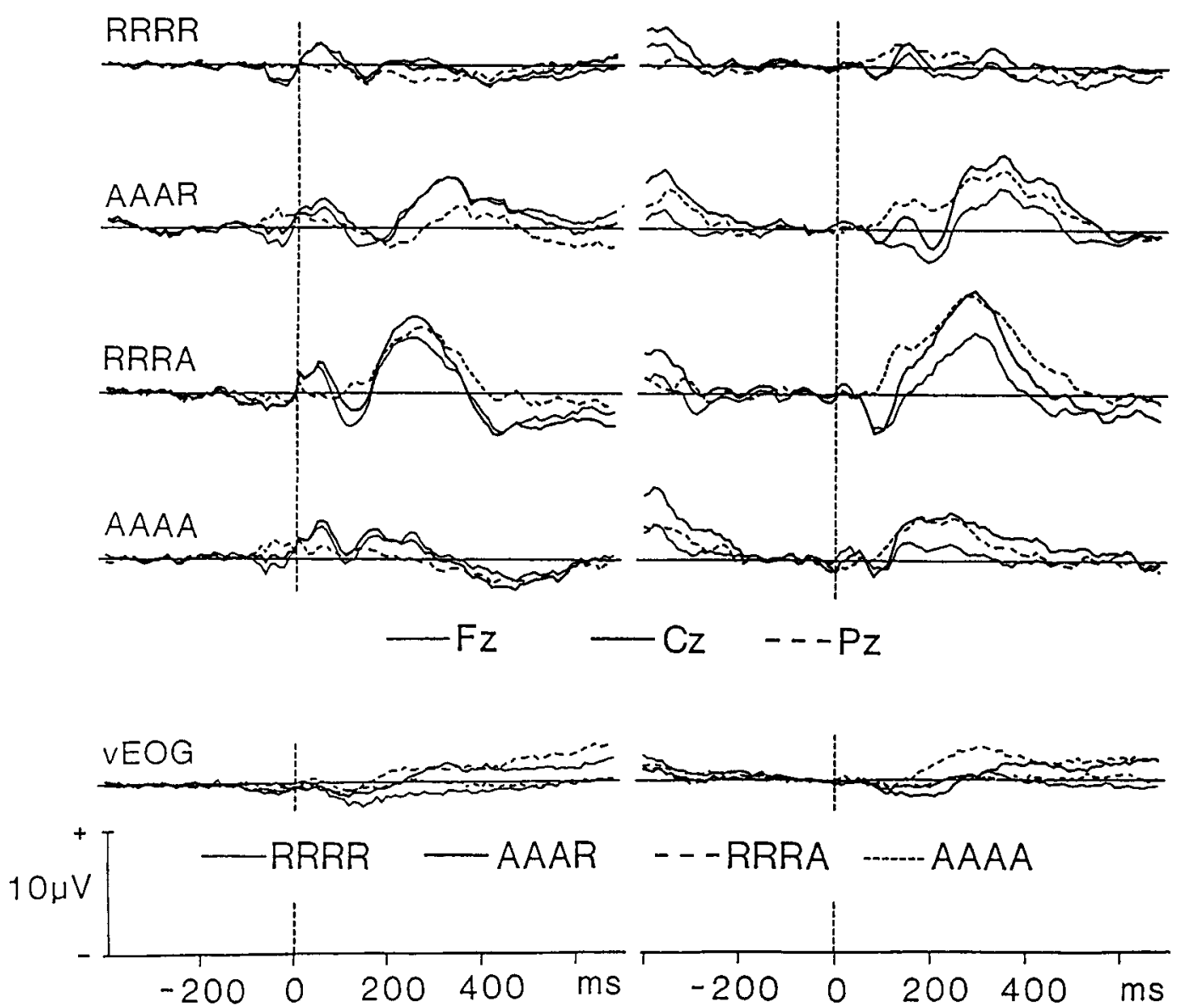

Figure 3. Experiment 1: Grand average event-related potentials (ERPs) with midline electrodes superimposed, and the vertical electrooculogram for the most extreme trial sequences. The left and right sides display ERPs from the 40- and 500-msec response-to-stimulus interval condition, respectively.

and the electrode site. In order to disambiguate such effects, we repeated the ANOVA after scaling the ERPs to the same overall amplitude across electrode sites within each condition. Scaling was performed with the average vector length across electrodes in the grand average for each condition.

The FO effect was highly significant $[F(1,9)=24.4$, $p<.001]$, with repetitions yielding smaller amplitudes than alternations $(M=1.2 \mathrm{vs} .2 .8 \mu \mathrm{V})]$. RSI did not alter this FO effect $(F<1)$. Again, the cost-benefit pattern was reflected by a strong FO $\times$ HO interaction $[F(1,9)=$ $73.0, p<.001]$, but now it was unmodulated by RSI $(F<1)$. The FO $\times$ HO interaction was present also when the RSI 40 was tested alone $[F(1,9)=25.0, p<.001]$. Note that there were no influences of RSI on sequential effects even in the interaction with the electrode. This held true also after scaling the amplitudes.
In contrast to the lack of modulations of sequential effects, RSI did affect P300 amplitude in general $[F(1,9)=$ $15.5, p<.01]$ and in interaction with electrode $[F(2,18)=$ $10.6, p<.01]$. An ANOVA on scaled amplitudes confirmed that reducing RSI from 500 to $40 \mathrm{msec}$ changed the common centroparietal distribution of $\mathrm{P} 300[M(\mathrm{Fz}$ to $\mathrm{Pz})=$ $1.7,4.3,3.4 \mu \mathrm{V}]$ to a frontocentral topography $[\mathrm{M}(\mathrm{Fz}$ to $\mathrm{Pz})=1.2,1.1,0.3 \mu \mathrm{V} ; F(2,18)=6.0, p<.05]$. In addition, in unscaled data, there were interactions of electrode with $\mathrm{FO}[F(2,18)=15.3, p<.01]$ and with both $\mathrm{FO}$ and $\mathrm{HO}[F(14,126)=2.7, p<.05]$. These interactions are probably due to the fact that amplitude changes at different electrodes are multiplicative rather than additive, because they disappeared after scaling for amplitude $(p \geq .1)$.

The sequential effects in P300 amplitude for the RSI 500 condition were mainly a replication of earlier results (Leuthold \& Sommer, 1993; Sommer et al., 1990a) for long 


\section{RSI 40ms}

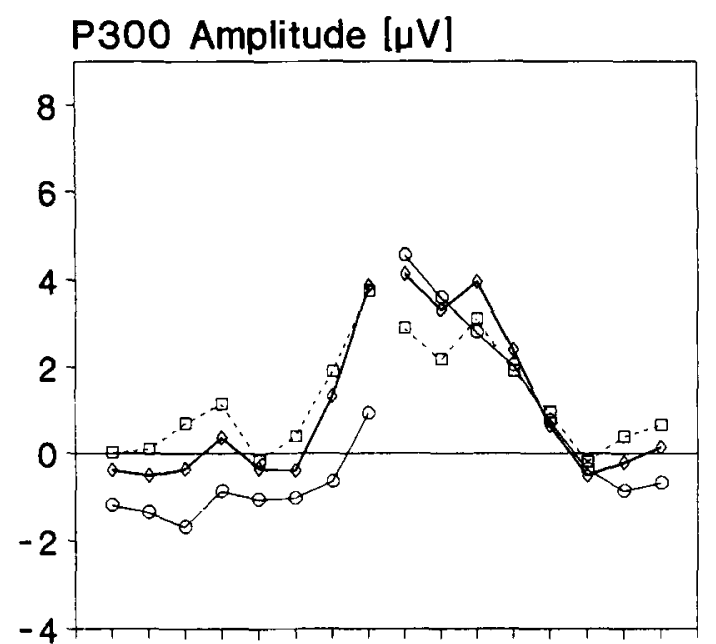

RARARARARARARARA RRAARRAARRAARRAA RRRRAAAARRRAAAA RRRRRRRRA A A A A A A
RSI 500ms

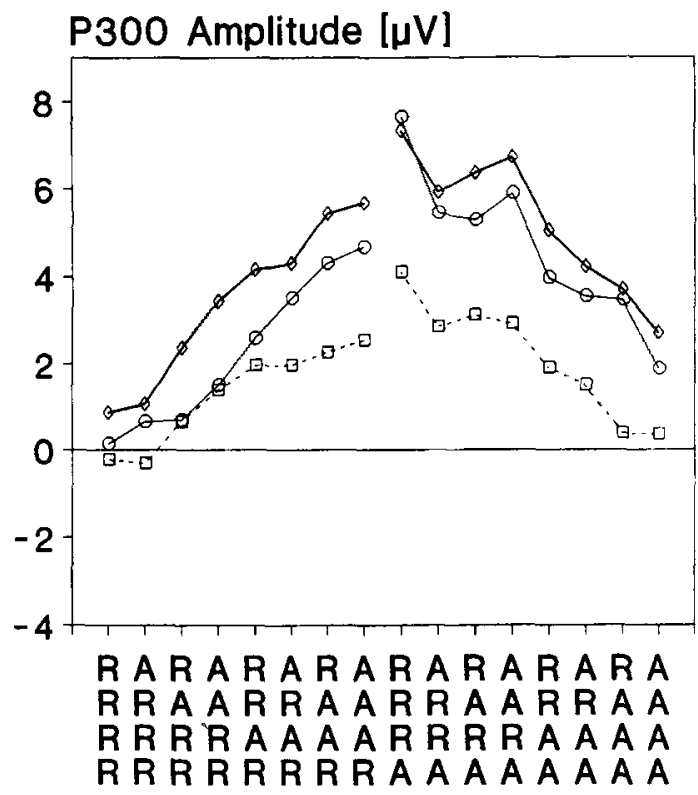

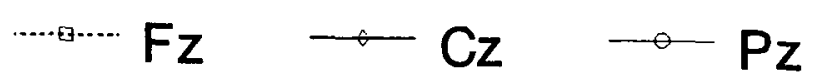

Figure 4. Experiment 1: Sequential effects in P300 amplitude at the midline electrodes. The left panel shows the RSI 40 condition after overlap correction, and the right panel shows the RSI 500 condition.

and constant ISIs, with HO effects in ERPs and RTs showing similar cost-benefit patterns. The present study, which defined the relevant stimulus in terms of the stimulated ear, extends earlier findings in which tone frequency was used to define the relevant stimulus.

The situation is different, however, in the RSI 40 condition. Whereas the alternation curve in RTs showed a highly significant change from a positive slope for RSI 40 to a negative slope for RSI 500, it remained negative across RSIs for P300 amplitude. This result is not an artifact of the overlap correction. First, the cost-benefit pattern also is visible for the extreme sequences in the uncorrected waveforms (cf. Figure 2). For these sequences, overlap by ERPs to preceding stimuli is a minor problem, because it concerns stimuli which elicit only small P300s. For example, an RRRA trial is preceded to equal parts by ARRR and RRRR stimuli, both of which elicit small ERPs. Second, even the uncorrected ERPs yielded similar results when analyzed in a way analogous to the corrected versions.

The dissociation between HO sequential effects in RTs and ERPs at short RSIs indicates that expectancies are present in these conditions but have no direct effect on behavior. Possibly these expectancies need a minimum time to develop into a preparatory state that can influence the RT process.

Whereas the general picture presented by the data of Experiment 1 conforms with previous results, there were no FO effects in the RTs. With visual stimuli, mostly an
FO repetition effect was found for short RSIs, and an FO alternation effect for long RSIs (see Kirby, 1980). Inasmuch as auditory localization tasks have not been used in previous studies of sequential effects, the specifics of this task may be responsible for our findings. To assess this hypothesis and to test the generalizability of our findings across modalities, we decided to repeat the experiment with a visual localization task.

\section{EXPERIMENT 2}

\section{Method}

Apart from the subjects and modality of stimulation, the method and instructions followed those of Experiment 1. Experiment 2 was performed with a new sample of 10 unpracticed right-handed subjects ( 3 males, 20 to 36 years of age). Stimulation was provided by three light-emitting diodes (LED) of 3-mm diameter, aligned horizontally at distances of $6 \mathrm{~mm}$ between their centers. Viewing distance was $1 \mathrm{~m}$. The middle LED was red and lit continuously, providing a fixation point and spatial reference. The outer LEDs were green and served as response signals when lit.

\section{Results and Discussion}

Performance. Unless stated otherwise, data analysis was conducted as in Experiment 1. Figure 5 depicts RTs and error rates for the two RSI conditions and the 16 trial sequences.

As in Experiment 1, responses were faster in the RSI 500 than in the RSI 40 condition $[M=313$ vs. $368 \mathrm{msec}$; $F(1,9)=20.9, p<.01]$. In contrast to Experiment 1 , there 


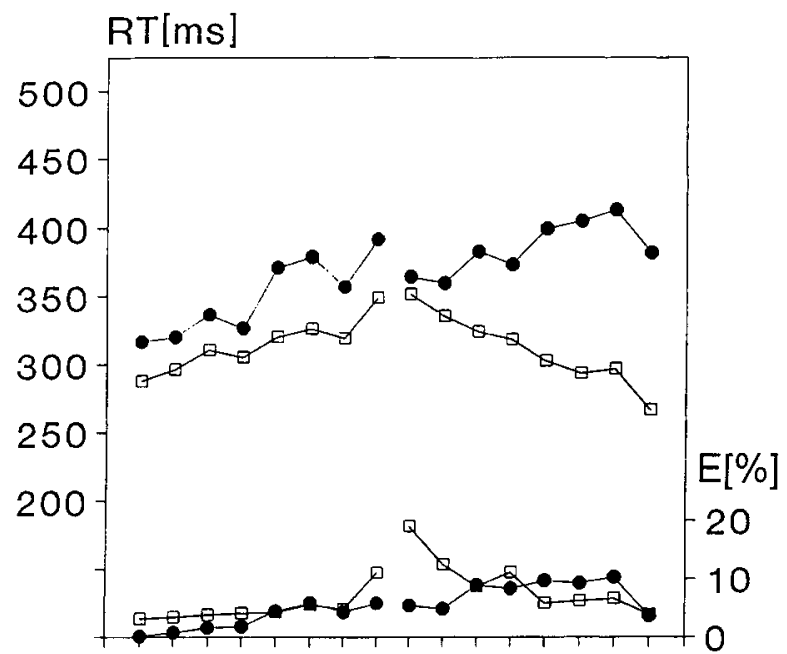

RARARARARARARARA RRAARRAARRAARRAA RRRRAAAARRRRAAAA RRRRRRRRAAAAAAA

\section{$\because \mathrm{RSI} 40 \mathrm{~ms} \rightarrow \mathrm{RSI} 500 \mathrm{~ms}$}

Figure 5. Experiment 2: Sequential effects in reaction times and error rates.

was a strong interaction of RSI and FO $[F(1,9)=40.1$, $p<.001]$, owing to a clear FO repetition effect for RSI 40 $[M=350$ vs. $385 \mathrm{msec} ; F(1,9)=11.4, p<.01]$, and a slight, nonsignificant tendency toward an alternation effect for RSI $500(M=314$ vs. $311 \mathrm{msec})$.

As before, the linear trends of the HO sequences depended on the FO sequence and the RSI condition $[F(1,9)$ $=21.8, p<.05]$. For the RSI 40 condition, slopes of both the repetition and the alternation branches were positive to a similar degree $(F=2.1)$. In contrast, the linear trends across the repetition and alternation branches in the RSI 500 condition differed widely in direction $[F(1,9)=212.6, p<.001]$. When the RSI conditions were compared for the repetition and alternation branches separately, they differed only little for the repetition branches $[F(1,9)=5.7, p<.05]$ but did so clearly for the alternation branches $[F(1,9)=23.0, p<.001]$.

As regards error rates, Experiment 2 confirmed the advantage of the short- over the long-RSI condition $[M=5.3 \%$ vs. $7.1 \% ; F(1,9)=5.2, p<.05]$. As before, more errors were made for FO alternations than for repetitions $[M=8.4 \%$ vs. $4.0 \% ; F(1,9)=10.4, p<.05]$. The HO sequential effects in error rates were similar to those in RTs. Specifically, there was a three-way RSI $\times$ FO $\times$ HO interaction $[F(1,9)=24.6, p<.001]$. This interaction relates to the inverted linear slopes across alternation and repetition sequences for RSI $500[F(1,9)=$ $49.0, p<.001]$, which are in contrast to the only slight differences in the positively sloped linear trends for RSI $40[F(1,9)=6.5, p<.05]$.
The results of the visual Experiment 2 mainly replicate those of the auditory Experiment 1, demonstrating the generalizability of the previous findings across modalities. However, whereas Experiment 1 did not yield any FO effects in RTs, these were as expected in Experiment 2 , with a repetition effect for RSI 40 . The absence of an FO alternation effect, which is usually found in visual, spatially compatible two-choice tasks with long RSIs (Kirby, 1972, 1976; Soetens et al., 1985), could be due to a practice effect. Indeed, the RSI 500 condition in the present study is always executed as the second session. Soetens et al. (1984) demonstrated that the FO alternation effect disappears with practice. The absence of an FO repetition effect at RSI 40 in Experiment 1 would appear to relate to stimulus factors. Although we cannot specify the critical properties, one could speculate that it relates to loudness adaptation during repeated stimulation of one ear, which creates a clear difference in sensation when the tone is switched to the other ear (cf. Dange, Warm, Weiler, \& Dember, 1993).

P300 amplitude. The same procedure of overlap correction as that used for the data of Experiment 1 was applied. Figure 6 shows midline ERPs and the EOG as a function of the extreme trial sequences; P300 amplitude was measured with the same procedure and is depicted for all sequences in Figure 7. As in Experiment 1, P300 amplitude shows the expected cost-benefit pattern for the HO sequences and the commonly observed FO repetition effect for both RSIs.

The ANOVA for P300 amplitudes confirmed the FO effect as being highly significant $[F(1,9)=31.6, p<$ $.001]$, with repetitions yielding smaller amplitudes than alternations ( $M=2.2$ vs. $3.9 \mu \mathrm{V})$. Again, the FO effect was independent of RSI $(F<1)$. As before, the costbenefit pattern was reflected by a strong $\mathrm{FO} \times \mathrm{HO}$ interaction $[F(1,9)=55.9, p<.001]$ unmodulated by RSI $(F<1)$. The FO $\times$ HO interaction was again present when RSI 40 was tested alone $[F(1,9)=42.4, p<.001]$. Note that, again, influences of RSI on sequential effects were not observed, which held true also for interactions with electrode or after scaling the ERP amplitudes.

Although RSI did not modulate sequential effects, it did affect $\mathrm{P} 300$ amplitude in general $[F(1,9)=171.8$, $p<.001]$ and in interaction with electrode $[F(2,18)=$ $35.0, p<.001$ ], as had been observed in Experiment 1 . Shortening RSI not only diminished P300 amplitude, it also changed its centroparietal distribution $[M(\mathrm{Fz}$ to $\mathrm{Pz})=$ $4.7,8.1,7.4 \mu \mathrm{V}]$ to a frontal topography $[M(\mathrm{Fz}$ to $\mathrm{Pz})=$ $-.4,-.9,-.7 \mu \mathrm{V}]$. An ANOVA on scaled data confirmed that the scalp topography was altered by RSI, independent of amplitude $[F(2,18)=7.7, p<.01]$. In addition, in unscaled data, there were interactions of electrode with $\mathrm{FO}[F(2,18)=13.6, p<.01]$ and with both $\mathrm{FO}$ and $\mathrm{HO}$ $[F(1,9)=38.9, p<.001]$. Again, these interactions seem to be mainly due to the fact that amplitude changes at different electrodes are not additive but multiplicative, because after scaling the interaction of electrode with FO 


\section{RSI 40ms}

RSI 500ms
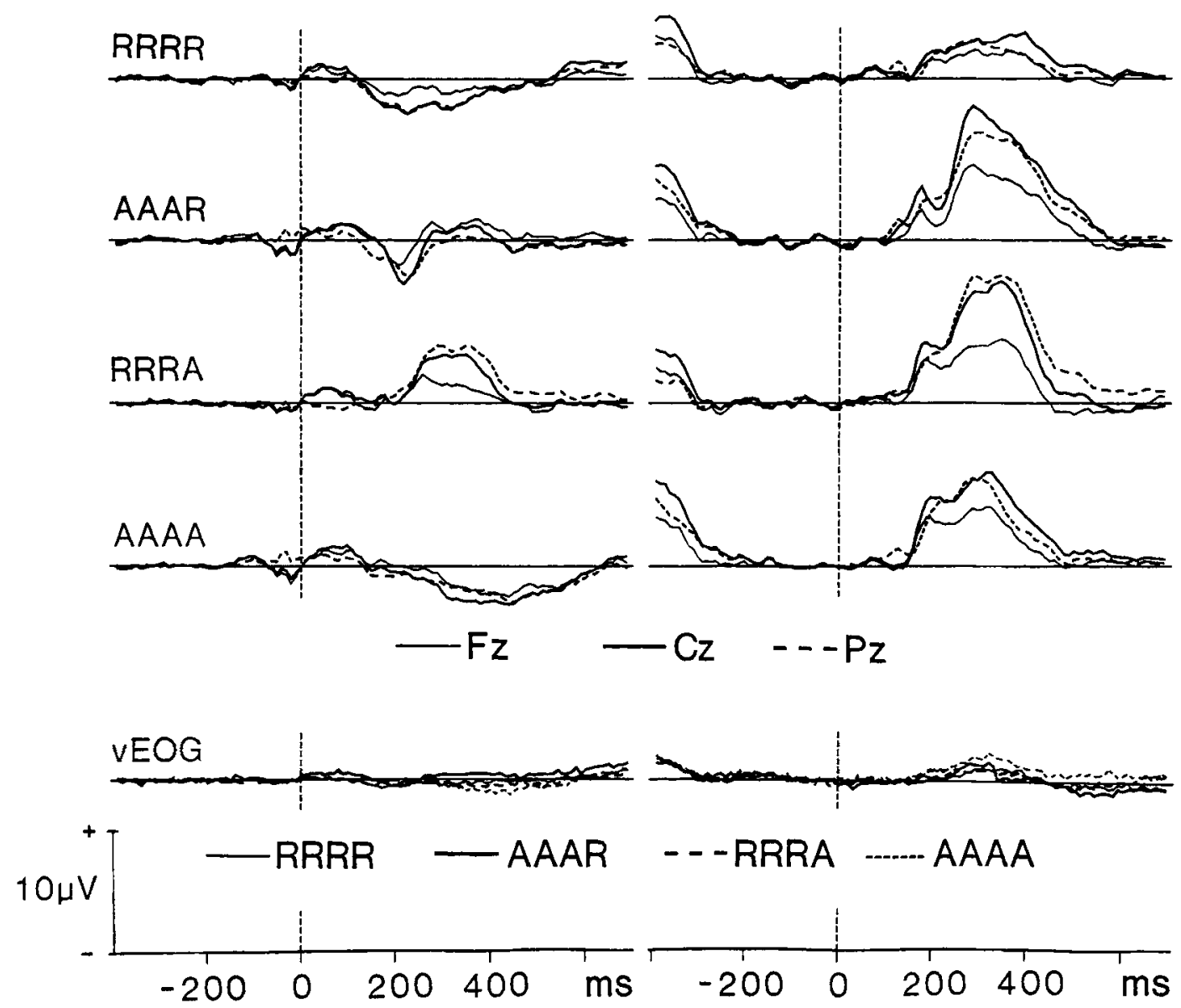

Figure 6. Experiment 2: Grand average event-related potentials (ERPs) with midline electrodes superimposed, and the vertical electrooculogram for the most extreme trial sequences. The left and right sides display ERPs from the 40- and 500-msec response-to-stimulus interval condition, respectively.

disappeared $(p=.1)$ and the interaction with $\mathrm{FO}$ and $\mathrm{HO}$ was greatly diminished $[F(1,9)=6.43, p<.05]$.

Sequential effects in P300 amplitude are in total agreement with Experiment 1, which is of particular interest in regard to the RSI 40 condition, where they have not been observed previously. Therefore, these findings have to be considered to be reliable.

\section{GENERAL DISCUSSION}

In serial two-choice RT experiments, two mechanisms appear to be responsible for the occurrence of sequential effects. Automatic facilitation has been found to dominate in conditions with short RSIs, whereas subjective expectancy dominates in conditions with long RSIs. The primary aim of the present contribution was to assess the relationship between these two mechanisms. By recording ERPs, we found support for the conjecture that, under both short- and long-RSI conditions, there is a buildup of expectancies that influences performance, however, only at long RSIs.

In contrast to the similarity of sequential effects between RTs and ERPs in conditions with long RSIs (Sommer et al., 1990a; Squires et al., 1976), different sequential effects were found with short RSIs. With short RSIs, RTs showed the typical benefit-only pattern of automatic facilitation, but $\mathrm{P} 300$ amplitude displayed the same costbenefit-like pattern of subjective expectancy as in longRSI conditions.

The observation of RSI-independent HO sequential effects in P300 amplitude lends support to the suggestion of Soetens et al. (1985; Soetens et al., 1984). They argued that expectancies may be present in all conditions, but at short RSIs they may not influence behavior. Therefore, a differentiation should be made between expectancy, on the one hand, and observable effects of these expectancies on behavior, on the other hand. This suggestion was based on exceptional RT effects on some 


\section{RSI $40 \mathrm{~ms}$}

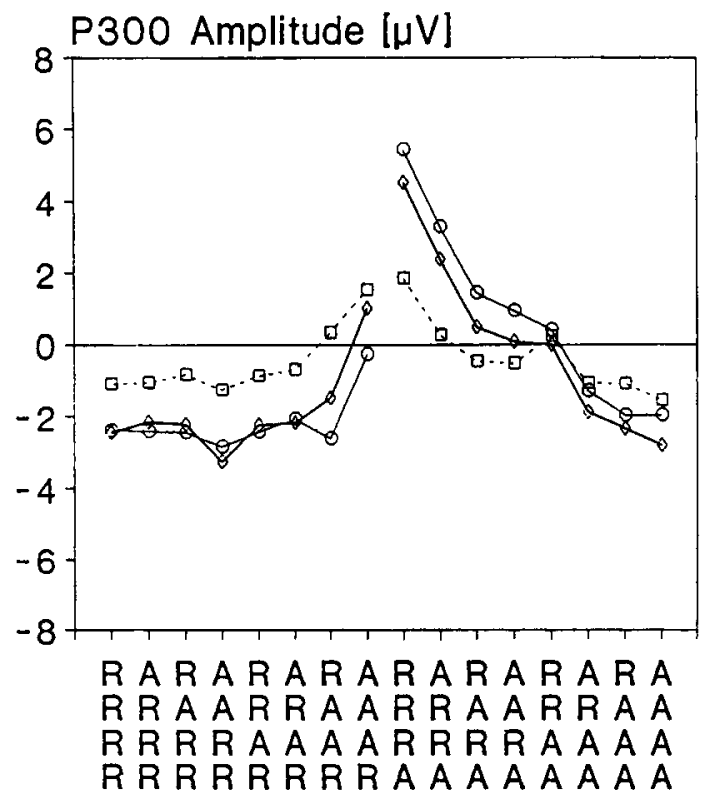

\section{RSI 500ms}

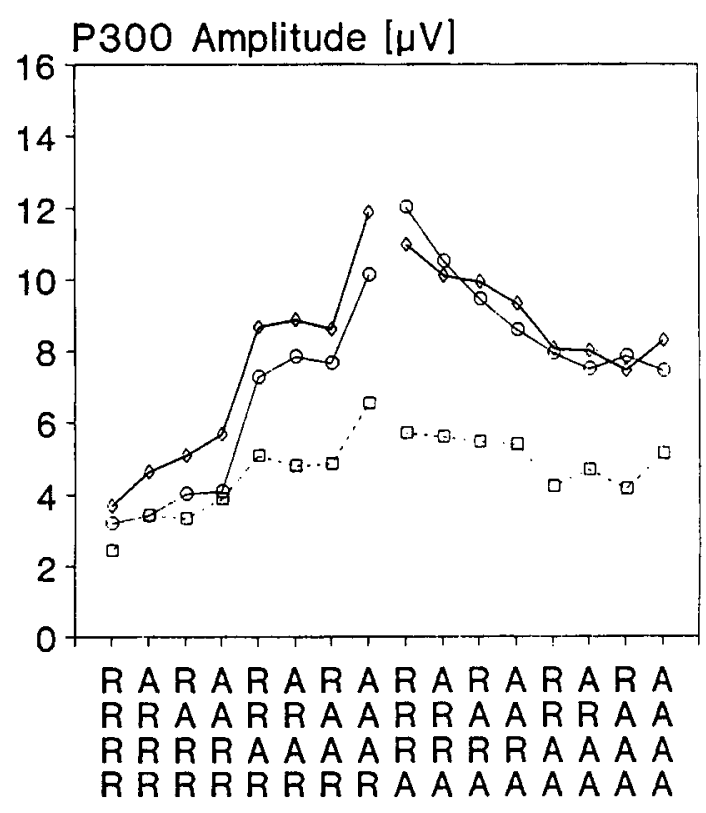

\section{$\cdots \mathrm{Cz} \longrightarrow \mathrm{Fz} \longrightarrow \mathrm{Pz}$}

Figure 7. Experiment 2: Sequential effects in P300 amplitude. The left panel shows the RSI 40-msec condition after overlap correction, and the right panel shows the RSI 500-msec condition.

stimulus sequences. The present results for ERP amplitudes show expectancy- typical cost-benefit patterns across the whole range of trial sequences, even at short RSIs, whereas at the behavioral level there was benefitonly, even without any sign of a breakthrough of expectancy. Therefore, we must conclude that expectancy is present at all RSIs but that, at short RSIs, automatic facilitation dominates RTs.

This interpretation is in accord with the idea that the expectancies in ERP amplitudes at long RSIs show some properties that are more of an automatic-like, rather than a controlled, nature and, as such, must be differentiated from expectancies related to higher cognitive processes (Johnson, 1988; Sommer et al., 1998). For instance, it has been shown that the $\mathrm{P} 300$ cost-benefit pattern is unconscious (Sommer et al., 1990a) and not influenced by probability manipulations (Matt et al., 1992). Finally, in the present data, the cost-benefit pattern observed in ERPs during long RSIs remains stable under a short-RSI condition, where this pattern is abolished for RTs. Although somewhat speculative, we consider it plausible that the critical factor for the switch from the cost-benefit to the benefit-only pattern in RTs relates to the availability of time. Because controlled processes are considered to be slow and time consuming (Shiffrin \& Schneider, 1977), one should expect a change in sequential effects if they are the product of a controlled process. The insensitivity of sequential effects in P300 amplitude toward time availability supports the idea that the process generating the cost-benefit pattern in $\mathrm{P} 300$ is not a controlled process in the strict sense. Therefore, the present findings from the RSI 40 condition add a further piece of evidence for the idea that the mechanism underlying the generation of sequential effects in P300 amplitude may be of a mixed nature. The properties of the subjective expectancy process clearly cuts across the classical distinction between automatic and controlled processing. This is no exceptional finding, since several automatic-like cost-benefit patterns have also been observed in priming studies (e.g., Neumann, 1990; Neumann \& Klotz, 1994).

In order to integrate our electrophysiological and behavioral findings, we assume that the sequential effects observed in $\mathrm{P} 300$ amplitude reflect a process that is responsible, in some sense, for sequential effects in RTs at long RSIs. We may, then, suggest the following mechanism. Expectancy, which is evoked in perceptual processes, feeds into the information-processing system at later, possibly response-related stages. This feed-forward system seems to need a minimum time, which is available in the RSI 500 condition but not in the RSI 40 condition. In the latter, the same expectancy patterns are present as those reflected in the P300 amplitude, but there is not enough time to influence the later stages. Support for such a minimum preparation time, based on expectancy, is found in discrete choice RT studies with variable fore- 
periods between an informative precue and the imperative stimulus. Sanders (1972), for example, found that at least $150-200 \mathrm{msec}$ were needed for the precue to have an effect on the RT. It is notable that this kind of mechanism can also explain why automatic facilitation survives over longer intervals when the RSI is short (HO effects over three to four stimuli), as compared with long RSIs. In the former, the sequential information that is supposed to influence response-related processes has to be updated with each intervening stimulus, so that its interaction with the RT process is prevented.

The absence of a benefit-only pattern in ERP results reveals some aspects of the interaction between both mechanisms. Pashler and Baylis (1991) claimed that the repetition effect is situated in the response selection stage, on the basis of their specific empirical data. Several other researchers have found evidence that automatic facilitation may be present in all processing stages (Rabbitt \& Vyas, 1973; Soetens et al., 1984), including the early perceptual stages (Maljkovic \& Nakayama, 1994, 1996). Because the low-level expectancy mechanisms also seem to be active in these early stages, as shown by the present ERP data, it can be assumed that both mechanisms are working in parallel, probably following different pathways. Following Sanders' (1977, 1980) idea of dividing information-processing mechanisms in those mechanisms related to the structural or functional pathways, we can associate automatic facilitation to the structural pathway, whereas expectancy mechanisms can be related to the functional pathway. Automatic facilitation effects can be regarded as working directly on the lowest level connection between stimulusand response-related nodes, more or less working according to the principle of connectionist networks, facilitating or inhibiting pathways under the influence of frequent use. Subjective expectancy effects, on the other hand, follow a different pathway, independent of the low-level connection between stimulus and response, making use of somewhat higher cognitive nodes, related to sequential structure. In agreement with the general assumption that the use of higher cognitive nodes (deeper processing) takes more time, it explains why this mechanism can only interfere in the low-level stream of information when enough time is available.

The suggestion that sequential effects in P300 amplitude are not caused by a controlled process may seem at odds with other findings that indicate that P300 amplitude reflects a controlled resource-consuming process. For example, P300 amplitude declines with perceptual load on secondary tasks (see, e.g., Sirevaag, Kramer, Coles, \& Donchin, 1989) and with practice (Rösler \& Manzey, 1986). However, this contradiction may be resolved by considering the sequence-independent changes in P300 with the RSI. There was a decrease in amplitude when the RSI was decreased from 500 to $40 \mathrm{msec}$, particularly at $\mathrm{Pz}$ and $\mathrm{Cz}$, leading to a change in scalp topography. However, this change in topography with the RSI did not interact with the FO or HO sequence, even when overall amplitude differences were controlled for by scaling. Therefore, the change appears to concern some aspect of P300 that is unrelated to the sequential effects. In other words, reducing the RSI removes a part of P300 that is present at long RSIs but that is unrelated to the trial sequence. What is left, then, at short RSIs may be a less concealed view of the sequence-dependent P300. This residual P300 has ascalp topography that is quite unlike the centroparietal P3b.

The results of the ANOVA on scaled P300 amplitudes also speak against a possible alternative interpretation of our findings: One might conceive that, at long RSIs, both a more frontal P300 and a separate parietal P300 component are influenced by the trial sequence in a similar vein. At short RSIs, the parietal P300 is diminished or eliminated, leaving mainly the frontal P300. In this case, one would expect not only a global reduction of P300 amplitude and a global change of scalp distribution, but also a change in scalp distribution as a function of the trial sequence. In other words, there should be an interaction of the trial sequence and electrode site with the RSI. However, nowhere in our data did such an interaction appear. The only interaction of electrode site and trial sequence that survived scaling for amplitude was observed in Experiment 2, where, independent of RSI, the $\mathrm{FO}$ and $\mathrm{HO}$ sequence interacted with electrode site. However, this interaction merely indicates that the generator structure of the P300 may differ between certain trial sequences and does not bear on the question of whether the RSI affects the generator configuration. Therefore, our data are rather more consistent with the idea that, both at long and at short RSIs, we are dealing with the same sequence-sensitive aspect of $\mathrm{P} 300$ and that the main difference between the two conditions lies in the contribution of a sequence-independent subcomponent of $\mathrm{P} 300$.

The sequence-sensitive component resembles the more frontal variants of $\mathrm{P} 300$, such as the $\mathrm{P} 3 \mathrm{a}$, elicited by very rare unattended stimuli (Ford, Roth, \& Kopell, 1976), or the novelty-P3 to singular events in the experimental context (Courchesne, Hillyard, \& Galambos, 1975). Common to these frontal variants of P300 is their involuntary elicitation. This converges with our suggestion that the frontal and sequence-dependent aspect of $\mathrm{P} 300$ is probably the product of a low-level (automatic) expectancy mechanism.

In conclusion, our ERP results suggest that the mechanism that is responsible for the expectancy effects in RTs at long RSIs is active also at short RSIs. Because we interpret the sequential effects in ERPs as being the product of an uncontrollable mechanism, it seems that this mechanism influences overt responses only when RSIs are of sufficient length.

\section{REFERENCES}

Audley, R. J. (1973). Some observations on theories of choice reaction time: Tutorial review. In S. Kornblum (Ed.), Attention and performance IV (pp. 509-545). New York: Academic Press.

BERTELSON, P. (1961). Sequential redundancy and speed in a serial two- 
choice responding task. Quarterly Journal of Experimental Psychologv, 13, 90-102.

BERTELSON, P. (1963). S-R relationships and reaction times to new versus repeated signals in a serial task. Journal of Experimental Psychology, 65, 478-484.

Bertelson, P., \& RENkin, A. (1966). Reaction times to new versus repeated signals in a serial task as a function of response-signal time interval. Acta Psychologica, 25, 132-136.

Campbell, K. C., \& Proctor, R. W. (1993). Repetition effects with categorizable stimulus and response sets. Journal of Experimental Psychology: Learning, Memory, \& Cognition, 19, 1345-1362.

Courchesne, E., Hillyard, S. A., \& Galambos, R. (1975). Stimulus novelty, task relevance and the visual evoked potential in man. Electroencephalography \& Clinical Neurophysiology, 39, 131-143.

Dange, A., WARM, J. S., Weiler, E. M., \& Dember, W. N. (1993). Loudness adaptation: Resolution of a psychophysical enigma. Journal of General Psychology, 120, 217-243.

Duncan-Johnson, C. C., Roth, W. T., \& Kopell, B. S. (1984). Effects of stimulus sequence on $\mathrm{P} 300$ and reaction time in schizophrenics. In R. Karrer, J. Cohen, \& P. Tueting (Eds.), Brain and information: Event-related potentials (Annals of the New York Academy of Sciences, Vol. 425, pp. 570-577). New York: New York Academy of Sciences.

Ford, J. M., Duncan-Johnson, C. C., Pfefferbaum, A., \& Kopell, B. S. (1982). Event-related potentials to a change of pace in a visual sequence. Psychophysiology, 19, 173-177.

Ford, J. M., RoTh, W. T., \& KopPELL, B. S. (1976). Auditory evoked potentials to unpredictable shifts in pitch. Psychophysiology, 13, 32-39.

HYMAN, R. (1953). Stimulus information as a determinant of reaction time. Journal of Experimental Psychology, 45, 188-196.

Johnson, R., JR. (1988). The amplitude of the P300 component of the event-related potential: Review and synthesis. In P. K. Ackles, J. R. Jennings, \& M. G. H. Coles (Eds.), Advances in psychophysiology (Vol. 3, pp. 69-138). Greenwich, CT: JAI Press.

KIRBY, N. H. (1972). Sequential effects in serial reaction time. Journal of Experimental Psychology, 96, 32-36.

KIRBY, N. H. (1976). Sequential effects in two-choice reaction time: Automatic facilitation of subjective expectancy. Journal of Experimental Psychology: Human Perception \& Performance, 2, 567-577.

KIRBY, N. H. (1980). Sequential effects in choice reaction time. In A. T. Welford (Ed.), Reaction times (pp. 129-172). London: Academic Press.

LAMING, D. R. J. (1968). Information theory of choice-reaction times. London: Academic Press.

LEUTHOLD, H., \& SOMMER, W. (1993). Stimulus presentation rate dissociates sequential effects in event-related potentials and reaction time. Psychophysiology, 30, 510-517.

Maljkovic, V., \& NaKaYAMa, K. (1994). Priming of pop-out: I. Role of features. Memory \& Cognition, 22, 657-672.

Maljkovic, V., \& Nakayama, K. (1996). Priming of pop-out: Il. The role of position. Perception \& Psychophysics, 58, 977-991.

Matt, J., Leuthold, H., \& Sommer, W. (1992). Differential effects of voluntary expectancies on reaction times and event-related potentials: Evidence for automatic and controlled expectancies. Journal of Experimental Psychology: Learning, Memory; \& Cognition, 18, 810-822.

MCCARThY, G., \& WoOD, C. C. (1985). Scalp distributions of eventrelated potentials: An ambiguity associated with analysis of variance models. Electroencephalography \& Clinical Neurophysiology, 62, 203-208.

NeUMANN, O. (1990). Direct parameter specification and the concept of perception. Psychological Research, 52, 207-215.

Neumann, O., \& KLotz, W. (1994). Motor responses to nonreportable, masked stimuli: Where is the limit of direct parameter specification? In C. Umiltà \& M. Moscovitch (Eds.), Attention and performance $X V$ : Conscious and nonconscious information processing (pp. 123-150). Cambridge, MA: MIT Press, Bradford Books.

Pashler, H., \& Baylis, G. (1991). Procedural learning: 2. Intertrial repetition effects in speeded-choice tasks. Journal of Experimental Psychology: Learning, Memory, \& Cognition, 17, 33-48.

PosNer, M. I., \& SNYDER, C. R. R. (1975). Facilitation and inhibition in the processing of signals. In P. M. A. Rabbitt \& S. Dornic (Eds.), Attention and performance V (pp. 669-682). London: Academic Press. RabiitT, P. M. A., \& VyAS, S. (1973). What is repeated in the "repetition effect"? In S. Kornblum (Ed.), Attention and performance $I V$ (pp. 327-342). New York: Academic Press.

RöSLER, F., \& MANZEY, D. (1986). Automatization of cognitive operations as reflected in event-related brain potentials: Methodological considerations and data. In F. Klix \& H. Hagendorf (Eds.), Human memory and cognitive capabilities (pp. 659-673). Amsterdam: Elsevier.

SANDERS, A. F. (1972). Foreperiod duration and the time course of preparation. Acta Psychologica, 36, 60-71.

SANDERS, A. F. (1977). Structural and functional aspects of the reaction process. In S. Dornic (Ed.), Attention and performance VI (pp. 3-25). Hillsdale, NJ: Erlbaum.

SANDERS, A. F. (1980). Stage analysis of reaction processes. In G. E. Stelmach \& J. Requin (Eds.), Tutorials in motor behavior (pp. 3-25). Amsterdam: North-Holland.

Schneider, W., \& Shiffrin, R. M. (1977). Controlled and automatic human information processing: I. Detection, search, and attention. Psychological Review, 84, 1-66.

SHIFFrIN, R. M., \& SchneIDER, W. (1977). Controlled and automatic human information processing: II. Perceptual learning, automatic attending, and a general theory. Psychological Review, 84, 127-190.

Sirevaag, E. J., Kramer, A. F., Coles, M. G. H., \& Donchin, E. (1989). Resource reciprocity: An event-related brain potentials analysis. Acta Psychologica, 70, 77-97.

SOETENS, E. (1990). Sequential effects in two-choice reaction time. Unpublished doctoral thesis, University of Leiden.

SOETENS, E. (1998). Localizing sequential effects in serial choice reaction time with the information reduction procedure. Journal of Experimental Psychology: Human Perception \& Performance, 24, 547568.

SoETENS, E., Boer, L. C., \& Hueting, J. E. (1985). Expectancy or automatic facilitation? Separating sequential effects in two-choice reaction time. Journal of Experimental Psychology: Human Perception \& Performance, 11, 598-616.

Soetens, E., Deboeck, M., \& Hueting, J. E. (1984). Automatic aftereffects in two-choice reaction time: A mathematical representation of some concepts. Journal of Experimental Psychology: Human Perception \& Performance, 10, 581-598.

Sommer, W., Leuthold, H., \& MATt, J. (1998). The expectancies that govern $\mathrm{P} 300$ amplitude are mostly automatic and unconscious. Behavioral \& Brain Sciences, 21, 149-150.

Sommer, W., MatT, J., \& LeUthold, H. (1990a). Consciousness of attention and expectancy as reflected in event-related potentials and reaction times. Journal of Experimental Psychology: Learning, Memory, \& Cognition, 16, 902-915.

Sommer, W., Matt, J., \& Leuthold, H. (1990b). Practice and sequential effects in reaction times and event-related potentials. In C. H. M. Brunia, A. W. K. Gaillard, \& A. Kok (Eds.), Psychophysiological brain research (pp. 26-31). Tilburg: Tilburg University Press.

Squires, K. C., Wickens, C., Squires, N. K., \& DonChin, E. (1976). The effect of stimulus sequence on the waveform of the cortical eventrelated potential. Science, 193, 1142-1146.

Vervaeck, K. R., \& BoeR, L. C. (1980). Sequential effects in two-choice reaction time: Subjective expectancy and automatic aftereffect at short response-stimulus intervals. Acta Psychologica, 44, 175-190.

WOLDORFF, M. G. (1993). Distortion of ERP averages due to overlap from temporally adjacent ERPs: Analysis and correction. Psychophysiology, 30, 98-119.

\section{NOTE}

1. We did not apply ADJAR to the RSI 500 condition because there was very little overlap of ERPs in this condition, rendering this correction procedure meaningless.

(Manuscript received December 13, 1996; revision accepted for publication January 10, 1998.) 